\title{
On Rad- $D_{12}$ Modules
}

\section{Yahya Talebi, Ali Reza Moniri Hamzekolaee and Derya Keskin Tütüncü}

\begin{abstract}
Let $M$ be a right $R$-module. We call $M$ Rad- $D_{12}$, if for every submodule $N$ of $M$, there exist a direct summand $K$ of $M$ and an epimorphism $\alpha: K \rightarrow M / N$ such that $K e r \alpha \subseteq \operatorname{Rad}(K)$. We show that a direct summand of a Rad- $D_{12}$ module need not be a Rad- $D_{12}$ module. We investigate completely Rad- $D_{12}$ modules (modules for which every direct summand is a Rad- $D_{12}$ module). We also show that a direct sum of Rad- $D_{12}$ modules need not be a Rad- $D_{12}$ module. Then we deal with some cases of direct sums of Rad- $D_{12}$ modules.
\end{abstract}

\section{Introduction}

Throughout this paper, we assume that all rings are associative with identity and all modules are unital right modules. Let $M$ be a module. The symbols, " $\leq$ ", "«" and "Rad $(M)$ " will denote a submodule, a small submodule and the Jacobson radical of $M$, respectively. The module $M$ is said to have $\left(D_{12}\right)$ (or is a $\left(D_{12}\right)$-module) if for every submodule $N$ of $M$, there exist a direct summand $K$ of $M$ and an epimorphism $\alpha: K \longrightarrow M / N$ such that $K e r \alpha \ll K$ (see [7]). In this paper we define $\operatorname{Rad}-D_{12}$ modules. The module $M$ is said to have $\operatorname{Rad}-D_{12}$ (or is a $\operatorname{Rad}-D_{12}$ module) if for every submodule $N$ of $M$, there exist a direct summand $K$ of $M$ and an epimorphism $\alpha: K \longrightarrow M / N$ such that $\operatorname{Ker} \alpha \subseteq \operatorname{Rad}(K)$. It is easy to see that every radical module $M$ (i.e.

Key Words: Small submodule, Rad- $\oplus$-supplemented module, Rad- $D_{12}$ modules, Duo module.

2010 Mathematics Subject Classification: Primary 16D10; Secondary 16P70.

Received: January, 2011.

Accepted: February, 2012. 
$\operatorname{Rad}(M)=M)$ is a $\operatorname{Rad}-D_{12}$ module. Therefore the $\mathbb{Z}$-module $\mathbb{Q}_{\mathbb{Z}}$ is $\operatorname{Rad}-D_{12}$, but it is not a $\left(D_{12}\right)$-module.

Let $M$ be a module. A submodule $N$ of $M$ is called a weak Rad-supplement (Rad-supplement) of a submodule $L$ of $M$ if $M=N+L$ and $N \cap L \subseteq \operatorname{Rad}(M)$ $(M=N+L$ and $N \cap L \subseteq \operatorname{Rad}(N))$. The module $M$ is called weakly Radsupplemented (Rad-supplemented) if every submodule $N$ of $M$ has a weak Rad-supplement (Rad-supplement). Rad-supplement submodule is defined in [13]. This new concept is also studied in [12] and [3]. According to [5], $M$ is called Rad- $\oplus$-supplemented if every submodule of $M$ has a Rad-supplement that is a direct summand of $M$.

In Section 2, we investigate some properties of $\operatorname{Rad}-D_{12}$ modules. We prove that the class of Rad- $D_{12}$ modules contains strictly the class of Rad$\oplus$-supplemented modules. In Section 3, we will be concerned with direct summands of Rad- $D_{12}$ modules. We provide a characterization of direct summands having Rad- $D_{12}$. Section 4 deals with direct sums of $\operatorname{Rad}-D_{12}$ modules. We show that a direct sum of $\operatorname{Rad}-D_{12}$ modules is $\operatorname{Rad}-D_{12}$ if the direct sum is a duo module.

\section{Rad- $D_{12}$ modules}

In this section we will show that the class of $\operatorname{Rad}-D_{12}$ modules contains properly the class of Rad- $\oplus$-supplemented modules.

Proposition 2.1. Let $M$ be a Rad- $\oplus$-supplemented module. Then $M$ is Rad$D_{12}$.

Proof. Let $N$ be a submodule of $M$. Since $M$ is $\operatorname{Rad}-\oplus$-supplemented, then there exist direct summands $K$ and $K^{\prime}$ of $M$ such that $M=N+K=K \oplus K^{\prime}$ and $N \cap K \subseteq \operatorname{Rad}(K)$. Now we have the epimorphism $g$ from $K$ to $M / N$ which is defined by $k \mapsto k+N$ with $\operatorname{Kerg}=N \cap K \subseteq \operatorname{Rad}(K)$. Hence $M$ is a $\operatorname{Rad}-D_{12}$ module.

Example 2.2. [7, Examples 4.5 and 4.6] Let $R$ be a local artinian ring with radical $W$ such that $W^{2}=0, Q=R / W$ is commutative, $\operatorname{dim}\left({ }_{Q} W\right)=2$ and $\operatorname{dim}\left(W_{Q}\right)=1$. Consider the indecomposable injective right $R$-module $U=[(R \oplus R) / D]$ with $W=R u+R v$ and $D=\{(u r,-v r) \mid r \in R\}$. By [7, Example 4.5], $U$ is not $\operatorname{Rad}-D_{12}$. Note that $U$ is Rad-supplemented. Now let $S=R / W$, the simple $R$-module, and $M=U \oplus S$. By [7, Example 4.6], $M$ is Rad- $D_{12}$, but not Rad- $\oplus$-supplemented. 
Example 2.3. Let $M=(\mathbb{Z} / p \mathbb{Z}) \oplus\left(\mathbb{Z} / p^{n} \mathbb{Z}\right)$ where $p$ is a prime number and $n$ is a nonzero positive integer. By [6, Corollary 1.6] and Proposition 2.1, $M$ is $\operatorname{Rad}-D_{12}$.

A module $M$ is called hereditary, if every submodule of $M$ is projective. Recall from [13] that a module $M$ is called generalized semiperfect if for every factor module of $M$, namely $M / N$, there exist a projective module $P$ and an epimorphism $f: P \longrightarrow M / N$ such that $\operatorname{Ker} f \subseteq \operatorname{Rad}(P)$. In this case $f$ is a generalized projective cover of $M / N$.

Theorem 2.4. The following are equivalent for a hereditary module $M$ :

(1) $M$ is generalized semiperfect;

(2) $M$ is $\operatorname{Rad}-D_{12}$;

(3) $M$ is Rad- $\oplus$-supplemented;

(4) $M$ is Rad-supplemented.

Proof. (1) $\Rightarrow$ (4) By [13, Proposition 2.1].

$(4) \Rightarrow(3)$ It is by [11, Lemma 2.1].

$(3) \Rightarrow(2)$ By Proposition 2.1.

$(2) \Rightarrow(1)$ Clear.

Let $M$ be a module and $U \leq M$. Then $U$ is called $Q S L$ in $M$ if $(A+U) / U$ is a direct summand of $M / U$, then there exists a direct summand $P$ of $M$ such that $P \leq A$ and $A+U=P+U$ (see [1]).

Proposition 2.5. Let $M$ be a weakly Rad-supplemented module with Rad $(M)$ QSL in $M$. Then $M$ is Rad- $D_{12}$.

Proof. Let $N \leq M$. Since $M$ is weakly Rad-supplemented, $(N+\operatorname{Rad}(M)) / \operatorname{Rad}(M)$ is a direct summand of $M / \operatorname{Rad}(M)$. Since $\operatorname{Rad}(M)$ is QSL in $M$, there exists a decomposition $M=K \oplus L$ such that $K \leq N$ and $N+\operatorname{Rad}(M)=K+\operatorname{Rad}(M)$. Now consider the epimorphism $\alpha: L \rightarrow M / N$ defined by $\alpha(l)=l+N(l \in L)$. It is easy to see that $\operatorname{Ker} \alpha \subseteq \operatorname{Rad}(L)$. Hence $M$ is $\operatorname{Rad}-D_{12}$.

Let $M$ be a module. We say that $M$ is $w$-local if $M$ has a unique maximal submodule. Clearly $M$ is w-local if and only if $\operatorname{Rad}(M)$ is maximal in $M$.

Lemma 2.6. Let $M$ be a Rad- $D_{12}$ module. If $\operatorname{Rad}(M) \neq M$, then $M$ has a nonzero w-local direct summand. 
Proof. Let $N$ be a maximal submodule of $M$. Then there exist a direct summand $K$ of $M$ and an epimorphism $\alpha: K \longrightarrow M / N$ such that $K e r \alpha \subseteq$ $\operatorname{Rad}(K)$. Clearly, $K \neq 0$ and $K e r \alpha$ is a maximal submodule of $K$. Therefore $\operatorname{Ker} \alpha=\operatorname{Rad}(K)$ and hence $K$ is a nonzero w-local direct summand of $M$.

Corollary 2.7. If $M$ is a Rad-D $D_{12}$ module with $\operatorname{Rad}(M) \ll M$, then $M$ contains a local direct summand.

Proof. Since $\operatorname{Rad}(M) \ll M, M$ is a $\left(D_{12}\right)$-module. Now apply the proof of Lemma 2.6.

\section{Direct summands of Rad- $D_{12}$ modules}

The following example exhibits a Rad- $D_{12}$ module that contains a direct summand which is not a Rad- $D_{12}$ module.

Example 3.1. Consider the right $R$-module $M=U \oplus S$ in Example 2.2. The module $M$ is $\operatorname{Rad}-D_{12}$, but the submodule $U$ is not $\operatorname{Rad}-D_{12}$.

Let $M$ be a module. We will say that $M$ is completely $\operatorname{Rad}-D_{12}$ if every direct summand of $M$ is $\operatorname{Rad}-D_{12}$.

Recall from [2] that a module $M$ is said to have $\left(P^{*}\right)$ property if for any submodule $N$ of $M$ there exists a direct summand $D$ of $M$ such that $D \subseteq N$ and $N / D \subseteq \operatorname{Rad}(M / D)$, equivalently, for every submodule $N$ of $M$ there exists a decomposition $M=K \oplus K^{\prime}$ such that $K \subseteq N$ and $N \cap K^{\prime} \subseteq \operatorname{Rad}\left(K^{\prime}\right)$. It is easy to check that every module with $\left(P^{*}\right)$ is Rad- $\oplus$-supplemented and hence Rad- $D_{12}$ by Proposition 2.1.

Proposition 3.2. A module with $\left(P^{*}\right)$ property is completely Rad-D $D_{12}$.

Proof. By [2, Lemma 16], every direct summand of a module with $\left(P^{*}\right)$ has $\left(P^{*}\right)$. Now the result follows from the fact that every module with $\left(P^{*}\right)$ is Rad- $D_{12}$. 
Example 3.3. (i) Let $F$ be a field and $R$ the upper triangular matrix ring $\left(\begin{array}{cc}F & F \\ 0 & F\end{array}\right)$. For submodules $A=\left(\begin{array}{cc}0 & F \\ 0 & F\end{array}\right)$ and $B=\left(\begin{array}{cc}F & F \\ 0 & 0\end{array}\right)$, let $M=A \oplus$ $(R / B)$. By [8, Lemma 3], $M$ has $\left(P^{*}\right)$. So by Proposition 3.2, $M$ is completely $\operatorname{Rad}-D_{12}$.

(ii) Let $M=\mathbb{Z}\left(p_{1}^{\infty}\right) \oplus \ldots \oplus \mathbb{Z}\left(p_{n}^{\infty}\right)$ where $p_{1}, \ldots, p_{n}$ are distinct prime integers. By [9, Example 2.16], $M$ has $\left(P^{*}\right)$. Hence $M$ is completely $\operatorname{Rad}-D_{12}$.

The converse of Proposition 3.2 is not true as we see in following example.

Example 3.4. Let $M$ be the $\mathbb{Z}$-module $\mathbb{Z} / 2 \mathbb{Z} \oplus \mathbb{Z} / 8 \mathbb{Z}$. Since $M$ is finitely generated, $M$ does not have $\left(P^{*}\right)$ by [8, Example 10]. By [6, Theorem 1.4], $M$ is $\oplus$-supplemented and hence Rad- $\oplus$-supplemented. By [10, Example 2.10], every direct summand of $M$ is $\oplus$-supplemented and hence $\operatorname{Rad}-\oplus$-supplemented. Therefore by Proposition 2.1, $M$ is completely Rad- $D_{12}$.

A module $M$ is called refinable if for any submodules $U, V$ of $M$ with $M=U+V$, there exists a direct summand $U^{\prime}$ of $M$ with $U^{\prime} \subseteq U$ and $M=U^{\prime}+V$ (see $\left.[4,11.26]\right)$. It is easy to prove that $M$ is refinable iff every submodule of $M$ is QSL.

Proposition 3.5. Let $M$ be a weakly Rad-supplemented refinable module. Then $M$ is $\operatorname{Rad}-D_{12}$.

Proof. By Proposition 2.5.

Corollary 3.6. Every weakly Rad-supplemented refinable module is completely Rad- $D_{12}$.

Proof. This is a consequence of Proposition 3.5 and the fact that every direct summand of a weakly Rad-supplemented refinable module is weakly Radsupplemented refinable.

Let $M$ be an $R$-module. By $P(M)$ we denote the sum of radical submodules of $M$.

Proposition 3.7. Let $M$ be a Rad-D $D_{12}$ module. If $P(M)$ is a direct summand of $M$, then $P(M)$ is a Rad-D $D_{12}$ module.

Proof. Let $M=P(M) \oplus L$ for some submodule $L$ of $M$. Let $X$ be a submodule of $P(M)$. By hypothesis, there exist a direct summand $K$ of $M$ and an epimorphism $\alpha: K \longrightarrow M /(X \oplus L)$ such that $K e r \alpha \subseteq \operatorname{Rad}(K)$. It is clear that $M /(X \oplus L) \cong P(M) / X$. Thus $\operatorname{Rad}(K / K e r \alpha)=K / K e r \alpha$, and so $\operatorname{Rad}(K)=K$. Therefore $K \subseteq P(M)$. This means that $P(M)$ is $\operatorname{Rad}-D_{12}$. 
The following result gives a new characterization of direct summands having $\operatorname{Rad}-D_{12}$.

Theorem 3.8. Let $M=M_{1} \oplus M_{2}$. Then $M_{2}$ is a Rad-D $D_{12}$ module if and only if for every submodule $N$ of $M$ containing $M_{1}$, there exist a direct summand $K$ of $M_{2}$ and an epimorphism $\varphi: M \longrightarrow M / N$ such that $K$ is a direct summand Rad-supplement of $\operatorname{Ker} \varphi$ in $M$.

Proof. Suppose that $M_{2}$ is a $\operatorname{Rad}-D_{12}$ module. Let $N \leq M$ with $M_{1} \subseteq N$. Consider the submodule $N \cap M_{2}$ of $M_{2}$. Then there exist a direct summand $K$ of $M_{2}$ and an epimorphism $\alpha: K \longrightarrow M_{2} /\left(N \cap M_{2}\right)$ such that Ker $\alpha \subseteq$ $\operatorname{Rad}(K)$. Note that $M=N+M_{2}$ and $K$ is a direct summand of $M$. Let $M=K \oplus K^{\prime}$ for some submodule $K^{\prime}$ of $M$. Consider the projection map $\eta: M \longrightarrow K$ and the isomorphism $\beta: M_{2} /\left(N \cap M_{2}\right) \longrightarrow M / N$ defined by $\beta\left(x+N \cap M_{2}\right)=x+N$. Thus $\beta \alpha \eta: M \longrightarrow M / N$ is an epimorphism. Let $\varphi=\beta \alpha \eta$. Clearly, we have $\operatorname{Ker} \varphi=\operatorname{Ker} \alpha \oplus K^{\prime}$. Therefore $M=K+\operatorname{Ker} \varphi$. Moreover $K \cap \operatorname{Ker} \varphi=\operatorname{Ker} \alpha \subseteq \operatorname{Rad}(K)$.

Conversely, suppose that every submodule of $M$ containing $M_{1}$ has the stated property. Let $H$ be a submodule of $M_{2}$. Consider the submodule $H \oplus M_{1}$ of $M$. By hypothesis, there exist a direct summand $K$ of $M_{2}$ and an epimorphism $\varphi: M \longrightarrow M /\left(H \oplus M_{1}\right)$ such that $M=K+\operatorname{Ker} \varphi$ and $K \cap \operatorname{Ker} \varphi \subseteq \operatorname{Rad}(K)$. Let $f: K \longrightarrow M /\left(H \oplus M_{1}\right)$ be the restriction of $\varphi$ to $K$. Consider the isomorphism $\eta: M /\left(H \oplus M_{1}\right) \longrightarrow M_{2} / H$ defined by $\eta\left(m_{1}+m_{2}+\left(H \oplus M_{1}\right)\right)=m_{2}+H$. Therefore $\eta f: K \longrightarrow M_{2} / H$ is an epimorphism. Let $\alpha=\eta f$. Clearly, $\operatorname{Ker} \alpha=\operatorname{Ker} f=K \cap K \operatorname{Ker} \varphi$. Thus $\operatorname{Ker} \alpha \subseteq \operatorname{Rad}(K)$. Hence $M_{2}$ is a $\operatorname{Rad}-D_{12}$ module.

\section{Direct sums of Rad- $D_{12}$ modules}

We begin this section by giving an example showing that the class of Rad- $D_{12}$ modules is not closed under direct sums.

Example 4.1. Let $R$ be a discrete valuation ring and let $K$ be its quotient field. There exist a free module $F$ and a submodule $X$ of $F$ such that $F / X \cong K$ since every module is a homomorphic image of a free module. Then $F$ is not Rad- $\oplus$-supplemented by [5, Example 2.15]. Since $R$ is a hereditary ring, then $F$ is hereditary. Therefore $F$ cannot be $\operatorname{Rad}-D_{12}$ from Theorem 2.4. Note that since $F \cong \bigoplus_{i \in I} R$ and $R$ is local, $F$ is a direct sum of $R a d$ - $D_{12}$-modules.

Let $M$ be a module. $M$ is called a duo module if every submodule of $M$ is fully invariant. We next give a sufficient condition for arbitrary direct sums of $\operatorname{Rad}-D_{12}$ modules to be $\operatorname{Rad}-D_{12}$. 
Theorem 4.2. Let $M=\bigoplus_{i \in I} M_{i}$ be a duo module. If each $M_{i}$ is Rad- $D_{12}$, then $M$ is Rad- $D_{12}$.

Proof. Let $L$ be a submodule of $M$. Since $M$ is a duo module we have $L=$ $\bigoplus_{i \in I}\left(L \cap M_{i}\right)$. Let $i \in I$. Because $M_{i}$ is Rad- $D_{12}$ and $L \cap M_{i}$ is a submodule of $M_{i}$, there exist a direct summand $K_{i}$ of $M_{i}$ and an epimorphism $\alpha_{i}$ : $K_{i} \rightarrow \frac{M_{i}}{L \cap M_{i}}$ with $\operatorname{Ker} \alpha_{i} \subseteq \operatorname{Rad}\left(K_{i}\right)$. Now we define the homomorphism $\alpha: \bigoplus_{i \in I} K_{i} \rightarrow \bigoplus_{i \in I}\left[\frac{M_{i}}{\left(L \cap M_{i}\right)}\right] \cong \frac{M}{\left[\bigoplus_{i \in I}\left(L \cap M_{i}\right)\right]}=\frac{M}{L}$ by $k_{i_{1}}+\ldots+k_{i_{n}} \mapsto$ $\alpha_{i_{1}}\left(k_{i_{1}}\right)+\ldots+\alpha_{i_{n}}\left(k_{i_{n}}\right)$ with $k_{i_{j}} \in K_{i_{j}}$ for every $j=1, \ldots, n$. It is not hard to check that $\alpha$ is an epimorphism with $\operatorname{Ker} \alpha \subseteq \operatorname{Rad}\left(\bigoplus_{i \in I} K_{i}\right)$ and $\bigoplus_{i \in I} K_{i}$ is a direct summand of $M$. It follows that $M$ is $\operatorname{Rad}-D_{12}$.

Recall that a module $M$ has Summand Intersection Property (SIP), if the intersection of any two direct summands of $M$ is again a direct summand of $M$. By [10, Page 969], every duo module has SIP.

Remark 4.3. Being duo module in Theorem 4.2 is not necessary. The module $M=\mathbb{Z} / 2 \mathbb{Z} \oplus \mathbb{Z} / 8 \mathbb{Z}$ in Example 3.4 is not a duo module ( $M$ doesn't have SIP). Also $\mathbb{Z} / 2 \mathbb{Z}, \mathbb{Z} / 8 \mathbb{Z}$ and $M$ are $\operatorname{Rad}-D_{12}$.

\section{References}

[1] M. Alkan, On $\tau$-lifting and $\tau$-semiperfect modules, Turkish J. Math. 33 (2009) 117-130.

[2] I. Al-Khazzi and P. F. Smith, Modules with chain condition on superfluous submodules, Comm. Algebra, 19(8) (1991) 2331-2351.

[3] E. Büyükaşik and C. Lomp, On a recent generalization of semiperfect rings, Bull. Aust. Math. Soc. 78 (2008) 317-325.

[4] J. Clark, C. Lomp, N. Vanaja and R. Wisbauer, Lifting Modules. Supplements and Projectivity in Module Theory, Frontiers in Mathematics, Birkhäuser, Basel-Boston-Berlin, 2006.

[5] Ş. Ecevit, M. T. Koşan and R. Tribak, Rad- $\oplus$-supplemented modules and cofinitely Rad- $\oplus$-supplemented modules, Algebra Colloq. 19(4) (2012) 637-648.

[6] A. Harmanci, D. Keskin and P. F. Smith, On $\oplus$-supplemented modules, Acta Math. Hungar. (83)(1-2) (1999) 161-169. 
[7] D. Keskin and W. Xue, Generalizations of lifting modules, Acta Math. Hungar. 91(3) (2001) 253-261.

[8] D. Keskin, Finite direct sums of $\left(D_{1}\right)$-modules, Turkish J. Math. 22(1) (1998) 85-91.

[9] D. Keskin, On lifting modules, Comm. Algebra, 28(7) (2000) 34273440.

[10] M. T. Koşan and D. Keskin Tütüncü, H-supplemented duo modules, J. Algebra Appl. 6(6) (2007) 965-971.

[11] E. Türkmen and A. Pancar, On radical supplemented modules, Int. J. Comput. Cognition 7(1) (2009) 62-64.

[12] Y. Wang and N. Ding, Generalized supplemented modules, Taiwanese J. Math. 10(6) (2006) 1589-1601.

[13] W. Xue, Characterizations of semiperfect and perfect rings, Publ. Mat. 40 (1996) 115-125.

Yahya Talebi, Ali Reza Moniri Hamzekolaee

Department of Mathematics,

University of Mazandaran,

Babolsar, Iran.

Email: talebi@umz.ac.ir

a.monirih@umz.ac.ir

Derya Keskin Ttnc,

Department of Mathematics,

University of Hacettepe,

06800 Beytepe, Ankara, Turkey.

Email: keskin@hacettepe.edu.tr 